\title{
Endothelin receptor A-specific stimulation of glomerular inflammation and injury in a streptozotocin-induced rat model of diabetes
}

\author{
M. A. Saleh • E. I. Boesen • J. S. Pollock • V. J. Savin • \\ D. M. Pollock
}

Received: 11 August 2010 / Accepted: 29 November 2010/Published online: 30 December 2010

(C) Springer-Verlag 2010

\begin{abstract}
Aims/hypothesis Activation of endothelin receptor-A $\left(\mathrm{ET}_{\mathrm{A}}\right)$ increases glomerular permeability to albumin $\left(\mathrm{P}_{\mathrm{alb}}\right)$ and elevates pro-inflammatory markers in hyperglycaemic rats. Methods Male Sprague-Dawley rats were given streptozotocin $(n=32)$ or saline (sham; $n=32)$. Half of the animals in each group received the $\mathrm{ET}_{\mathrm{A}}$-selective antagonist, $\mathrm{ABT}-627$ (atrasentan; orally), beginning immediately after hyperglycaemia was confirmed. Glomeruli were isolated by sieving techniques and $\mathrm{P}_{\mathrm{alb}}$ determined from the change in glomerular volume induced by oncotic gradients of albumin. Glomerular nephrin levels were assessed by immunofluorescence, whereas urinary nephrin was measured by immunoassay.
\end{abstract}

M. A. Saleh · E. I. Boesen · J. S. Pollock • D. M. Pollock $(\bowtie)$

Vascular Biology Center, Medical College of Georgia,

1459 Laney Walker Blvd,

Augusta, GA 30907-2500, USA

e-mail: dpollock@mcg.edu

M. A. Saleh · J. S. Pollock • D. M. Pollock

Department of Pharmacology and Toxicology,

Medical College of Georgia,

Augusta, GA, USA

E. I. Boesen · D. M. Pollock

Department of Physiology, Medical College of Georgia,

Augusta, GA, USA

D. M. Pollock

Department of Surgery, Medical College of Georgia,

Augusta, GA, USA

V. J. Savin

Department of Medicine, Kansas City VAMC,

Kansas City, MO, USA
Results At 3 and 6 weeks after streptozotocin injection, proteinuria was significantly increased compared with sham controls and significantly reduced by ABT- 627 treatment. $\mathrm{P}_{\text {alb }}$ was also increased at 3 and 6 weeks post-streptozotocin. ABT-627 had no effect on $\mathrm{P}_{\text {alb }}$ or protein excretion in sham control rats. In glomeruli isolated from hyperglycaemic rats, incubation with BQ-123, a selective $\mathrm{ET}_{\mathrm{A}}$ antagonist, reduced $\mathrm{P}_{\mathrm{alb}}$, whereas $\mathrm{BQ}-788$, a selective endothelin receptor-B antagonist had no effect ( $n=6$ rats per group, 5-8 glomeruli per rat). Glomerular and plasma content of soluble intercellular adhesion molecule-1 and monocyte chemoattractant protein-1 were significantly increased 6 weeks after streptozotocin (ELISA). ABT-627 attenuated these increases. After 6 weeks of hyperglycaemia, glomerular nephrin content was decreased with a concurrent increase in urinary nephrin excretion. ABT-627 prevented glomerular nephrin loss in hyperglycaemic rats $(n=5-8$ rats per group; eight groups).

Conclusions/interpretation These observations support the hypothesis that endothelin-1, via the $\mathrm{ET}_{\mathrm{A}}$ receptor, directly increases $\mathrm{P}_{\mathrm{alb}}$, possibly via nephrin loss, as well as early inflammation in the hyperglycaemic rat.

Keywords Albuminuria - Diabetic nephropathy . Endothelin-1 · Glomerular permeability · MCP-1 · Nephrin · $\mathrm{P}_{\mathrm{alb}} \cdot \mathrm{sICAM}-1$

$\begin{array}{ll}\text { Abbreviations } \\ \mathrm{ET}_{\mathrm{A}} & \text { Endothelin receptor-A } \\ \mathrm{ET}_{\mathrm{B}} & \text { Endothelin receptor-B } \\ \mathrm{ICAM}-1 & \text { Intercellular adhesion molecule-1 } \\ \mathrm{MCP}-1 & \text { Monocyte chemoattractant protein-1 } \\ \mathrm{P}_{\mathrm{alb}} & \text { Glomerular permeability to albumin } \\ \text { PMSF } & \text { Phenylmethylsulfonylfluoride }\end{array}$




\section{Introduction}

Diabetes is the leading cause of end-stage renal disease in the western world, affecting approximately $30 \%$ of type 1 diabetic patients [1]. Microalbuminuria is the earliest clinical marker of renal complications in diabetes [2]. Patients develop macroproteinuria as renal injury progresses and suffer from GFR reduction, which subsequently leads to renal failure.

In addition to vascular endothelium, components of the endothelin-1 system are located in many cell types in the kidney, including mesangial cells [3], podocytes [4] and tubular epithelium [5]. Aside from vasoconstriction [6], endothelin-1 is a potent proinflammatory and profibrotic mediator [7]. In diabetes, intrarenal endothelin-1 activity appears to be increased [8]. Glomerular endothelin-1 mRNA expression is elevated in rats with streptozotocin-induced diabetes [9]. Kidney endothelin receptor-A $\left(\mathrm{ET}_{\mathrm{A}}\right)$ expression is upregulated in rabbits with alloxan-induced diabetes [10]. Consistent with these observations is the finding that urinary endothelin-1 excretion (a marker of intrarenal endothelin-1 generation) is increased in diabetic patients with microalbuminuria [11]. Furthermore, our laboratory has demonstrated that endothelin-1 plasma levels were not changed, but endothelin1 excretion was increased in rats after streptozotocin-induced hyperglycaemia [12].

Studies from several labs including our own have shown that chronic blockade of $\mathrm{ET}_{\mathrm{A}}$ reduces albuminuria and renal inflammation in the streptozotocin-induced rat model of diabetes, along with a modest decrease [12] or no significant change [13] in arterial pressure. However, the mechanism for this effect is not known. Inflammation is considered to be a contributing factor in the development of diabetic nephropathy and may contribute to proteinuria as well as interstitial fibrosis and cellular damage. Proinflammatory chemokines monocyte chemoattractant protein-1 (MCP-1) and soluble intercellular adhesion molecule-1 (ICAM-1) are important in attracting infiltrating cells and inducing their attachment to the endothelium, thus facilitating the early process of macrophage infiltration into the kidney [14]. Increased ICAM-1 expression leading to increased leucocyte infiltration has been demonstrated in experimental models of nephropathy [15].

Proteinuria represents an early sign of glomerular injury, the presence of which predicts not only an elevated risk of nephropathy, but also cardiovascular disease in general [16]. The mechanistic pathways of proteinuria in diabetes have not been fully resolved. One of the glomerular filtration barrier defects leading to glomerular injury and subsequent proteinuria is damage to the filtration-slit formed by glomerular podocytes. Nephrin, a 1241-residue transmembrane immunoglobulin protein, is an important filtration-slit molecule [17]. Levels and urinary excretion of nephrin show characteristic changes in diabetes and in other acquired proteinuric diseases [18].

The present study was undertaken to test the hypothesis that $\mathrm{ET}_{\mathrm{A}}$ contribute to glomerular inflammation and permeability defects in a rat model of hyperglycaemia by directly impairing glomerular permeability and promotion of glomerular inflammation. More specifically, we investigated whether endothelin-1 via the $\mathrm{ET}_{\mathrm{A}}$ has a direct effect on the glomerulus, thus inducing nephrin shedding and reducing glomerular permeability to albumin $\left(\mathrm{P}_{\mathrm{alb}}\right)$, which would provide a mechanism for diabetic proteinuria. We also investigated whether the anti-inflammatory actions of $\mathrm{ET}_{\mathrm{A}}$ blockade were due to changes in expression of early inflammatory response pathways such as MCP-1 and soluble ICAM-1. We used an in vivo model to specifically address these questions in the streptozotocin-induced rat model of type 1 diabetes.

\section{Methods}

Streptozotocin-induced hyperglycaemia Experiments used male Sprague-Dawley rats (250-275 g) from Harlan Laboratories (Indianapolis, IN, USA). All protocols were approved by the Institutional Animal Care and Use Committee of the Medical College of Georgia and followed the NIH Public Health Service Policy on Humane Care and Use of Laboratory Animals. Rats were housed in constant temperature and humidity, and exposed to a $12 \mathrm{~h}$ light-dark cycle. We studied four groups of rats: (1) sham-injected controls; (2) hyperglycaemic; (3) sham+ABT-627 (5 mg kg ${ }^{-1}$ day $^{-1}$, in drinking water); and (4) hyperglycaemic +ABT-627 ( $n=8$ in all groups). Hyperglycaemia was attained by injection of streptozotocin (Sigma-Aldrich, St Louis, MO, USA) at a dose of $65 \mathrm{mg} / \mathrm{kg}$ body weight, given intravenously through the penile vein under isoflurane anaesthesia; sham-injected animals received saline. ABT-627 is a selective $\mathrm{ET}_{\mathrm{A}}$ blocker (around 1000 times greater affinity for $\mathrm{ET}_{\mathrm{A}}$ vs endothelin receptor- $\mathrm{B}\left[\mathrm{ET}_{\mathrm{B}}\right]$ ) and provides maximum $\mathrm{ET}_{\mathrm{A}}$ blockade and selectivity at this dose in vivo [19]. Drug treatment was started 1 day after streptozotocin injection and after confirming that all rats had blood glucose levels $>20 \mathrm{mmol} / \mathrm{l}$. At the same time, insulin or palmitic acid (blank) implants (Linshin, Scarborough, ON, Canada) were inserted subcutaneously into the hyperglycaemic and shaminjected rats, respectively. Insulin implants maintained blood glucose levels at 20 to $25 \mathrm{mmol} / \mathrm{l}$ (Table 1). Glucose in whole blood (taken from a small incision on the tail) was measured with a glucometer (Accu-Chek; F. Hoffmann-La Roche AG, Basel, Switzerland). Glycaemia was monitored twice a week throughout the study. During the final 2 days of treatment, rats were placed in metabolism cages in order 
Table 1 Characteristics of the experimental rats after 3 and 6 weeks of treatment

\begin{tabular}{lllll}
\hline Characteristic & \multicolumn{2}{l}{ Animal group } & & \\
\cline { 2 - 5 } & Sham & Sham+ABT-627 & $\mathrm{HG}^{\text {a }}$ & HG+ABT-627 \\
\hline At 3 weeks & & & $280 \pm 10^{*}$ & $282 \pm 7^{*}$ \\
Body weight (g) & $311 \pm 5$ & $313 \pm 11$ & $22.2 \pm 0.6^{*}$ & $23.1 \pm 0.6^{*}$ \\
Blood glucose (mmol/l) & $5.6 \pm 0.4$ & $5.7 \pm 0.6$ & $42 \pm 4^{*}$ & $42 \pm 4^{*}$ \\
Food intake (g/day) & $23 \pm 4$ & $18 \pm 2$ & $160 \pm 21^{*}$ & $152 \pm 11^{*}$ \\
Water intake (ml/day) & $32 \pm 2$ & $30 \pm 2$ & $145 \pm 12^{*}$ & $141 \pm 10^{*}$ \\
Urine flow (ml/day) & $15 \pm 2$ & $18 \pm 3$ & & \\
At 6 weeks & & & $301 \pm 6^{*}$ & $303 \pm 9^{*}$ \\
Body weight (g) & $409 \pm 13$ & $412 \pm 13$ & $22.7 \pm 0.7^{*}$ & $22.9 \pm 0.9^{*}$ \\
Blood glucose (mmol/l) & $5.5 \pm 0.5$ & $5.7 \pm 0.6$ & $40 \pm 2^{*}$ & $38 \pm 3^{*}$ \\
Food intake (g/day) & $20 \pm 3$ & $18 \pm 2$ & $195 \pm 18^{*}$ & $187 \pm 15^{*}$ \\
Water intake (ml/day) & $29 \pm 3$ & $28 \pm 3$ & $170 \pm 15^{*}$ & $163 \pm 11^{*}$ \\
Urine flow (ml/day) & $15 \pm 1$ & $19 \pm 2$ & & \\
\hline
\end{tabular}

Data are means $\pm \operatorname{SEM}(n=8$ in all groups)

${ }^{\text {a}}$ Hyperglycaemic (HG) rats had partial insulin replacement to limit the degree of hyperglycaemia

${ }^{\mathrm{b}}$ Data derived from $24 \mathrm{~h}$ urine collections in metabolism cages ${ }^{*} p<0.05$ vs sham groups pepstatin) and homogenised by ultrasonic homogeniser (20 s). After centrifugation for $10 \mathrm{~min}$ at $10,000 \mathrm{~g}$, the supernatant fraction was used for analysis and protein determined using the Bradford method (Bio-Rad Laboratories, Hercules, CA, USA) according to the manufacturer's instructions.

Measurement of $P_{\text {alb }}$ After isolation, glomeruli were resuspended at room temperature in 5\% (wt/vol.) BSA (containing $115 \mathrm{mmol} / \mathrm{l} \mathrm{NaCl}, 5 \mathrm{mmol} / \mathrm{K} \mathrm{KCl}, 10 \mathrm{mmol} / \mathrm{l}$ sodium acetate, $1.2 \mathrm{mmol} / \mathrm{l}$ dibasic sodium phosphate, $25 \mathrm{mmol} / 1$ sodium bicarbonate, $1.2 \mathrm{mmol} / 1$ magnesium sulphate, $1 \mathrm{mmol} / 1$ calcium chloride and $3.5 \mathrm{mmol} / 1$ glucose, $\mathrm{pH} 7.4$ ).

The rationale and methodology for determination of albumin permeability has been described in detail previously [21]. In brief, images of 10-15 glomeruli per kidney preparation (i.e. per rat) were captured using a digital camera through an inverted microscope before and after a medium change to one containing $1 \%$ (wt/vol.) BSA. The medium exchange created an oncotic gradient across the basement membrane resulting in capillary expansion and a glomerular volume increase $\left(\Delta V=\left[V_{\text {final }}-V_{\text {initial }}\right] / V_{\text {initial }}\right)$, which was measured off-line by an image analysis programme (Digimizer; MedCalc Software, Mariakerke, Belgium). The software determined the average radius of the glomerulus in two-dimensional space and the volume was then derived from the formula $V=(4 / 3) \pi \mathrm{r}^{3}$. The magnitude of $\Delta V$ was related to the albumin reflection coefficient, $\sigma_{\text {alb }}$, by the following equation: $\left(\sigma_{\mathrm{alb}}\right)$ experimental $=(\Delta V)$ experimental $/$ $(\Delta V)_{\text {control; }}$ the $\sigma_{\text {alb }}$ of the control glomeruli was assumed to be equal to $1 . \mathrm{P}_{\mathrm{alb}}$ was defined as $1-\sigma_{\mathrm{alb}}$, and describes the movement of albumin subsequent to water flux. When $\sigma_{\text {alb }}$ is zero, albumin moves across the membrane with the same velocity as water and $\mathrm{P}_{\mathrm{alb}}$ is 1.0. Conversely, when $\sigma_{\mathrm{alb}}$ is 1.0 , albumin cannot cross the membrane with water and $\mathrm{P}_{\mathrm{alb}}$ is zero. vanadate, $1 \mathrm{mmol} / \mathrm{l} \mathrm{PMSF}, 1 \mu \mathrm{g} / \mathrm{ml}$ leupeptin and $1 \mu \mathrm{g} / \mathrm{ml}$ 
In additional experiments, we examined the role of $\mathrm{ET}_{\mathrm{A}}$ and $\mathrm{ET}_{\mathrm{B}}$ on $\mathrm{P}_{\mathrm{alb}}$ in glomeruli isolated from untreated hyperglycaemic rats $(n=6)$ using a selective $\mathrm{ET}_{\mathrm{A}}$ antagonist (BQ-123; Calbiochem, San Diego, CA, USA) and a selective $\mathrm{ET}_{\mathrm{B}}$ antagonist (BQ-788; Calbiochem). Glomeruli were pre-incubated with these antagonists at concentrations of $1 \times 10^{-9}$ to $1 \times 10^{-5} \mathrm{~mol} / 1$ for $15 \mathrm{~min}$ at $37^{\circ} \mathrm{C}$ and the $\mathrm{P}_{\text {alb }}$ response was determined. These experiments used a minimum of five glomeruli from each rat.

Biochemical analyses Commercially available kits for soluble ICAM-1 (Quantikine sICAM-1 Immunoassay; R\&D Systems, Minneapolis, MN, USA) and MCP-1 (RayBioTech, Norcross, GA, USA) were used to determine concentrations in plasma and glomerular homogenates. Nephrin concentration was determined in urine via an ELISA kit (Exocell, Philadelphia, PA, USA). Urinary protein concentrations were determined using the Bradford method.

Nephrin immunofluorescence Isolated glomeruli taken from kidneys perfused with PBS were placed on engraved glass slides and allowed to dry at room temperature before freezing at $-80^{\circ} \mathrm{C}$. Glomeruli were then fixed using paraformaldehyde $(2 \%$ vol./vol. $)$ in a slide chamber (Antibody Amplifier; IHC World, LLC, Woodstock, MD, USA ). Slides were then washed with PBS and incubated for $1 \mathrm{~h}$ with normal goat serum (5\% vol./vol.) in PBS and Triton-X ( $0.3 \%$ vol./vol.) before being incubated overnight on a shaker at $4{ }^{\circ} \mathrm{C}$ with goat anti-human nephrin primary antibody (1:500 vol./vol.; sc-19000; Santa Cruz Biotechnology, Santa Cruz, CA, USA). On the second day, washing and blocking procedures were repeated before incubating slides for $1 \mathrm{~h}$ in the antibody amplifier chamber with a mixture of Alexa Fluor 488 chicken anti-goat IgG fluorescent-tagged secondary antibody (1:5,000 vol./vol.) and rhodamine phalloidin for F-actin staining $(5 \mu \mathrm{mol} / \mathrm{l})$. Both antibodies were purchased from Invitrogen (Carlsbad, CA, USA). Washing was repeated and slides mounted with glass coverslips. Images were acquired with the confocal/ multiphoton system (FV-1000 MPE; Olympus, Center Valley, PA, USA) available at the Indiana Center for Biological Microscopy (Indianapolis, IN, USA). Confocal image stacks of whole glomeruli were collected with a $60 \times$ W NA1. 2 objective at $512 \times 512$ frame size and sequential mode using 488 and $559 \mathrm{~nm}$ laser lines. Nephrin and actin signals were collected with the emission filter at 520 and $612 \mathrm{~nm}$, respectively. At least five glomeruli per rat were imaged. Metamorph software version 7.5 (Molecular Devices, Downingtown, PA, USA) was used for quantitative analysis. A sum of all planes intensities for both channels within a Z-stack was used to measure the total intensity values within the glomerulus. Background values from control stacks were subtracted from the total intensity values for both channels. Nephrin signals were then normalised to the corresponding actin signal by calculating the ratio from the background-corrected total intensity values.

Statistical analyses All data are presented as mean \pm SEM. Differences between data obtained from sham, sham + ABT627 , hyperglycaemic and hyperglycaemic + ABT-627 animals were compared using two-way ANOVA followed by Bonferroni post hoc tests. Differences between 3- and 6-week-old groups were compared by using the unpaired Student's $t$ test. A value of $p<0.05$ was considered statistically significant. Analyses were performed using GraphPad Prism Version 5.0 software (GraphPad Software, La Jolla, CA, USA).

\section{Results}

As expected, blood glucose levels were elevated in hyperglycaemic rats with or without $\mathrm{ET}_{\mathrm{A}}$ blockade (Table 1). Similarly, blood glucose in sham-injected rats was not changed by treatment with ABT-627. Relative to sham and sham +ABT-627 rats, rats in the hyperglycaemic and hyperglycaemic + ABT-627 groups had lower body weights during the 3- and 6-week periods of study despite having hyperphagia, polydipsia and polyuria. Treatment with ABT627 did not change any of these variables.

Glomerular permeability and proteinuria $\mathrm{P}_{\mathrm{alb}}$ significantly increased after 3 weeks of hyperglycaemia when compared with sham. Treating animals with ABT-627 significantly decreased the elevated $\mathrm{P}_{\mathrm{alb}}$ value. $\mathrm{P}_{\mathrm{alb}}$ further increased significantly after 6 weeks of hyperglycaemia. Again, ABT627 significantly decreased $\mathrm{P}_{\text {alb }}$ after 6 weeks of treatment (Fig. 1a). Changes in urinary protein excretion followed a similar pattern to that of $\mathrm{P}_{\text {alb }}$. As shown in Fig. 1b, rats with hyperglycaemia exhibited significant proteinuria at 3 weeks compared with sham-injected rats. ABT-627 reduced proteinuria to levels comparable to sham. Proteinuria was further increased after 6 weeks of hyperglycaemia. Again, ABT- 627 prevented the increase in proteinuria at the 6 week time-point.

To further determine the role of glomerular $\mathrm{ET}_{\mathrm{A}}$ and $\mathrm{ET}_{\mathrm{B}}$ in regulating $\mathrm{P}_{\mathrm{alb}}$ in hyperglycaemic rats, isolated glomeruli from hyperglycaemic rats were incubated for $15 \mathrm{~min}$ at $37^{\circ} \mathrm{C}$ with soluble endothelin-1 antagonist peptides. At $10^{-7} \mathrm{~mol} / \mathrm{l}$, the $\mathrm{ET}_{\mathrm{A}}$ antagonist, $\mathrm{BQ} 123$, significantly reduced the elevated $\mathrm{P}_{\text {alb }}$ (Fig. 2a). $\mathrm{P}_{\text {alb }}$ was further reduced at higher $\mathrm{BQ} 123$ concentrations. In contrast, the $\mathrm{ET}_{\mathrm{B}}$ antagonist, BQ788, had no effect on the elevated $\mathrm{P}_{\mathrm{alb}}$ of hyperglycaemic glomeruli at any concentration of that antagonist (Fig. 2b). Incubation of isolated glomeruli from hyperglycaemic rats with $\mathrm{BQ} 123$ and $\mathrm{BQ} 788$ reduced $\mathrm{P}_{\mathrm{alb}}$ in a fashion similar to BQ123 alone (Fig. 2c). 


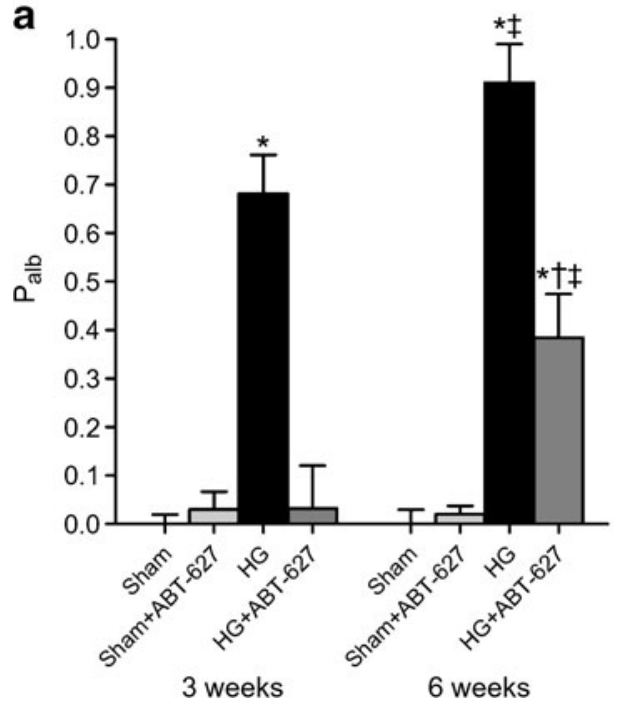

Fig. 1 Effect of streptozotocin-induced hyperglycaemia (HG), with or without concurrent treatment with ABT-627, on $\mathrm{P}_{\text {alb }}$ (a) and daily protein excretion rate (b) at times indicated; $n=5-8$ per group. Two-way ANOVA resulted in the following $p$ values: $\mathrm{P}_{\mathrm{alb}}, 3$ weeks, $p_{\mathrm{HG}}=0.0001$, $p_{\mathrm{ABT}-627}=0.0003, p_{\mathrm{HG} \times \mathrm{ABT}-627}=0.0002$ and 6 weeks, $p_{\mathrm{HG}}<0.0001$,

Systemic and glomerular inflammation Soluble ICAM-1 and MCP-1 are two early pro-inflammatory molecules implicated in the pathogenesis of diabetic nephropathy. After 3 weeks of hyperglycaemia, we did not observe any changes in plasma or glomerular levels of soluble ICAM-1 and MCP-1 (Fig. 3a, b, Fig. 4a, b). After 6 weeks, however, plasma and glomerular soluble ICAM-1 and MCP-1 were significantly elevated compared with those in sham-injected animals. These increases were significantly attenuated by ABT-627.

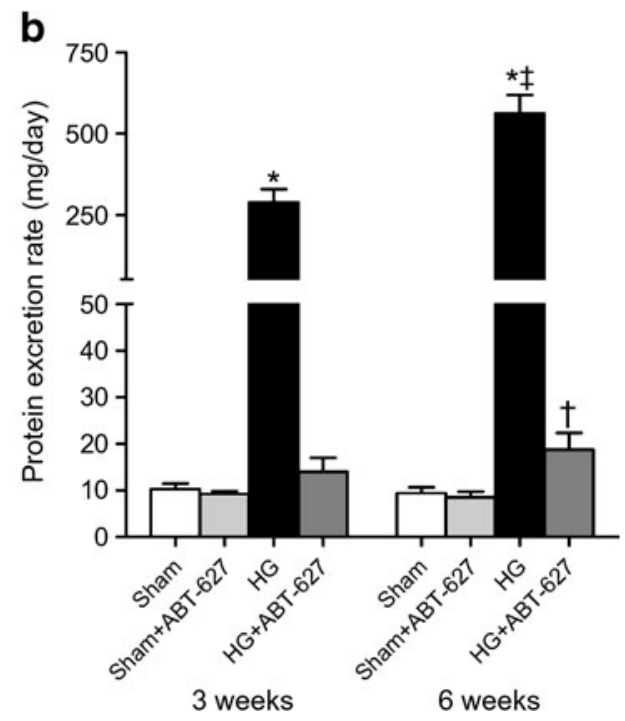

$p_{\mathrm{ABT}-627}=0.0001, p_{\mathrm{HG} \times \mathrm{ABT}-627}<0.0001$; protein excretion rate, 3 and 6 weeks, $p_{\mathrm{HG}}<0.0001, p_{\mathrm{ABT}-627}<0.0001, p_{\mathrm{HG} \times \mathrm{ABT}-627}<0.0001$. Post hoc analysis comparing individual means are denoted by $* p<0.05$ vs sham group, ${ }^{\dagger} p<0.05$ vs hyperglycaemia, and ${ }^{\star} p<0.05$ vs 3 -week treatment

Nephrinuria and glomerular nephrin expression We monitored glomerular levels of nephrin by immunostaining using fluorescence microscopy. As depicted in Fig. 5, glomeruli isolated from 6-week hyperglycaemic rats exhibited significantly lower nephrin levels than those of sham (Fig. 6a). In addition, glomeruli isolated from ABT627-treated hyperglycaemic rats had nephrin levels comparable to those of sham-injected animals (Fig. 6a). Nephrin is also excreted into urine in the early stages of diabetic nephropathy, which is an early sign of breakdown of the
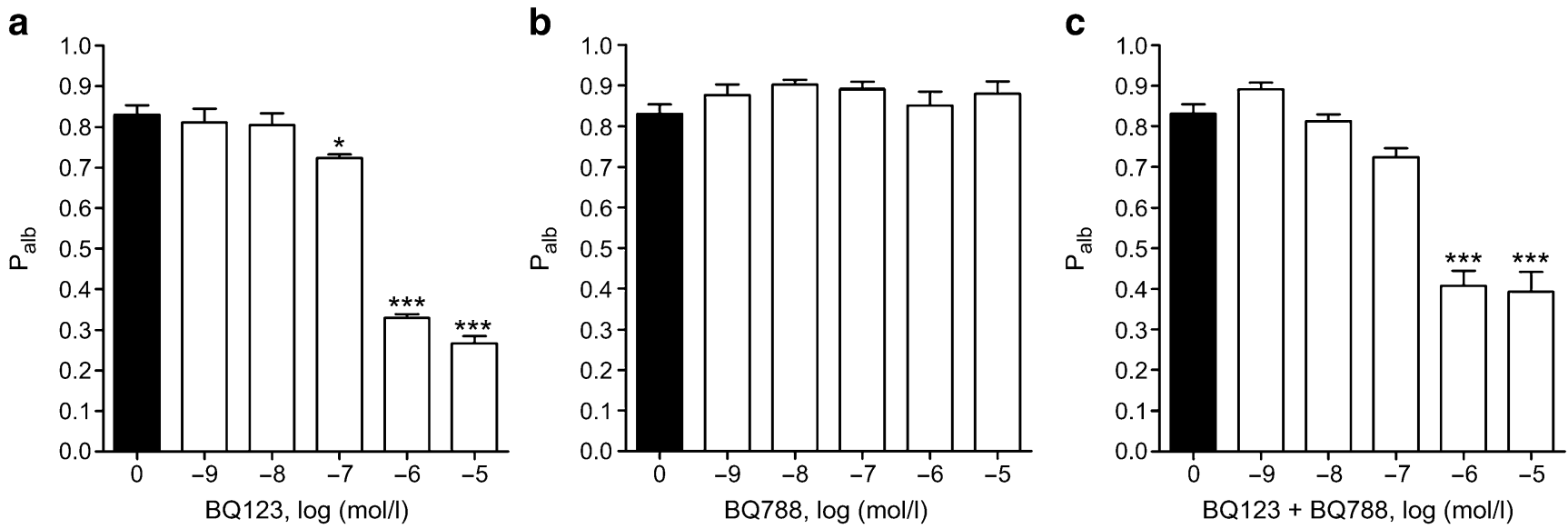

Fig. 2 In vitro effect of selective $\mathrm{ET}_{\mathrm{A}}$ and $\mathrm{ET}_{\mathrm{B}}$ antagonists on $\mathrm{P}_{\mathrm{alb}}$ of isolated glomeruli from untreated hyperglycaemic rats after incubation with concentrations ranging from $1 \times 10^{-9}$ to $1 \times 10^{-5} \mathrm{~mol}$ for $15 \mathrm{~min}$ at $37^{\circ} \mathrm{C}$. a BQ123 $\left(1 \times 10^{-5}\right.$ to $\left.1 \times 10^{-9} \mathrm{~mol} / \mathrm{l}\right)$ significantly reduced the elevated $\mathrm{P}_{\mathrm{alb}}$ of glomeruli isolated at 6 weeks of hyperglycaemia. b
BQ788 had no effect on elevated $\mathrm{P}_{\text {alb }}$ of hyperglycaemic glomeruli. c Addition of both antagonists produced the same effect as that of BQ123 alone. Hyperglycaemic rats used for treatment of glomeruli in vitro: $n=6$; at least five glomeruli per rat were analysed. ${ }^{*} p<0.05$, $* * * p<0.001$ vs zero concentration 
Fig. 3 Effect of streptozotocininduced hyperglycaemia (HG), with or without concurrent treatment with ABT-627, on (a) plasma soluble ICAM-1 (sICAM-1) and (b) plasma MCP-1 concentrations at times indicated; $n=5-8$ per group. Two-way ANOVA resulted in the following $p$ values: plasma soluble ICAM-1, 6 weeks, $p_{\mathrm{HG}}=0.016, p_{\mathrm{ABT}-627}=0.033$, $p_{\mathrm{HG} \times \mathrm{ABT}-627}<0.0001$; plasma MCP-1, 6 weeks, $p_{\mathrm{HG}}=0.0001$, $p_{\text {ABT }-627}=0.0056, p_{\mathrm{HG} \times \mathrm{ABT}-627}=$ 0.0008 . Post hoc analysis comparing individual means are denoted by $* p<0.05$ vs sham group, ${ }^{\dagger} p<0.05$ vs hyperglycaemia, and ${ }^{\star} p<0.05$ vs 3-week treatment a

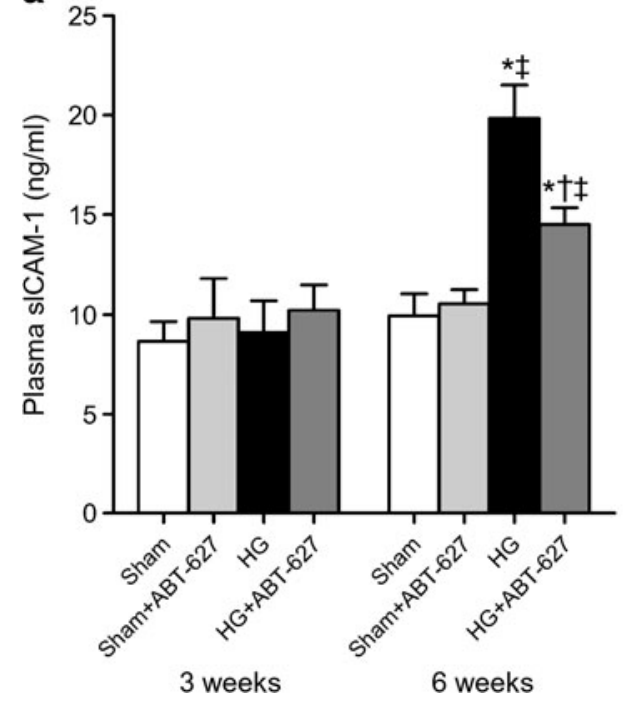

b

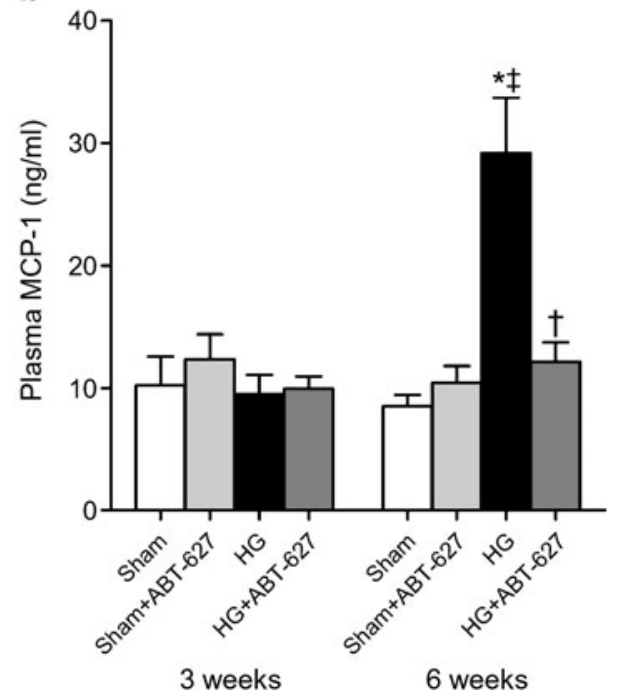

glomerular filtration barrier. Therefore, we determined whether nephrin loss in urine of hyperglycaemic rats is $\mathrm{ET}_{\mathrm{A}}$-dependent. As expected, urinary excretion of nephrin was significantly increased after 6 weeks of hyperglycaemia, while ABT-627 administration completely prevented the hyperglycaemia-induced increase in nephrin excretion (Fig. 6b).

\section{Discussion}

The current study provides new information on the mechanisms of endothelin effects in the glomerulus of the hyperglycaemic kidney and the potential use of endothelin antagonists in proteinuric renal disease. This includes evidence of a direct, $\mathrm{ET}_{\mathrm{A}}$-dependent increase in glomerular permeability and nephrin loss that occurs in the hyperglycaemic kidney. In addition, $\mathrm{ET}_{\mathrm{A}}$ blockade provides antiinflammatory actions by reducing hyperglycaemiadependent increases in early inflammatory markers such as MCP-1 and soluble ICAM-1. With the increased incidence of chronic diabetic complications, strategies able to improve prevention of end-stage renal disease are urgently needed. Experimental data suggest that endothelin receptor blockade could be a novel therapeutic approach to end-stage renal disease [12], while results from clinical trials suggest that these drugs could, in addition, be used in conditions such as diabetic nephropathy [22]. The protective effects of endothelin receptor antagonists on renal injury appear to be independent of blood pressure lowering.

The potential role of direct glomerular actions of endothelin receptors has not been defined. We observed
Fig. 4 Effect of streptozotocininduced hyperglycaemia (HG), with or without concurrent treatment with ABT-627, on (a) glomerular soluble ICAM-1 (sICAM-1) and (b) glomerular MCP-1 concentrations at times indicated; $n=5-8$ per group. Two-way ANOVA resulted in the following $p$ values: glomerular soluble ICAM-1, 6 weeks, $p_{\mathrm{HG}}<0.0001, p_{\mathrm{ABT}-627}=0.0153$, $p_{\mathrm{HG} \times \mathrm{ABT}-627}=0.0279$; glomerular MCP-1, 6 weeks,

$p_{\mathrm{HG}}<0.0001, p_{\text {ABT-627 }}=0.0097$, $p_{\mathrm{HG} \times \mathrm{ABT}-627}=0.0342$. Post hoc analysis comparing individual means are denoted by ${ }^{*} p<0.05$ vs sham, ${ }^{\dagger} p<0.05$ vs hyperglycaemia and ${ }^{\star} p<0.05$ vs 3-week treatment a

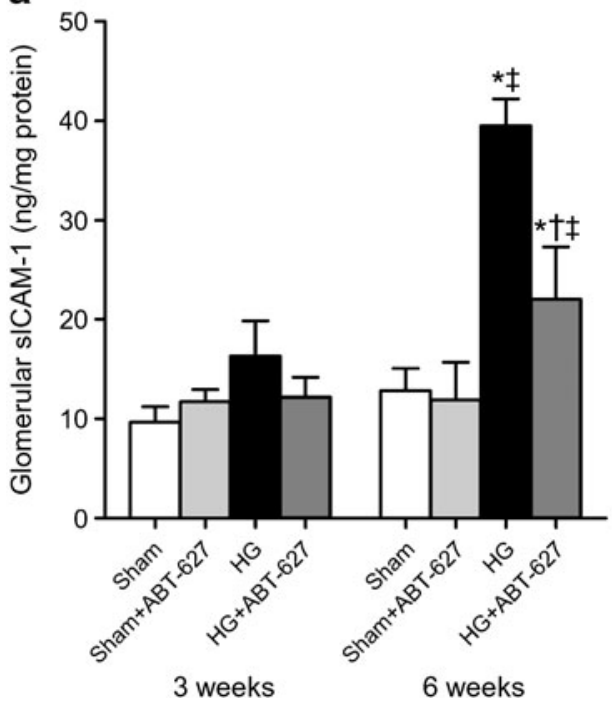

b

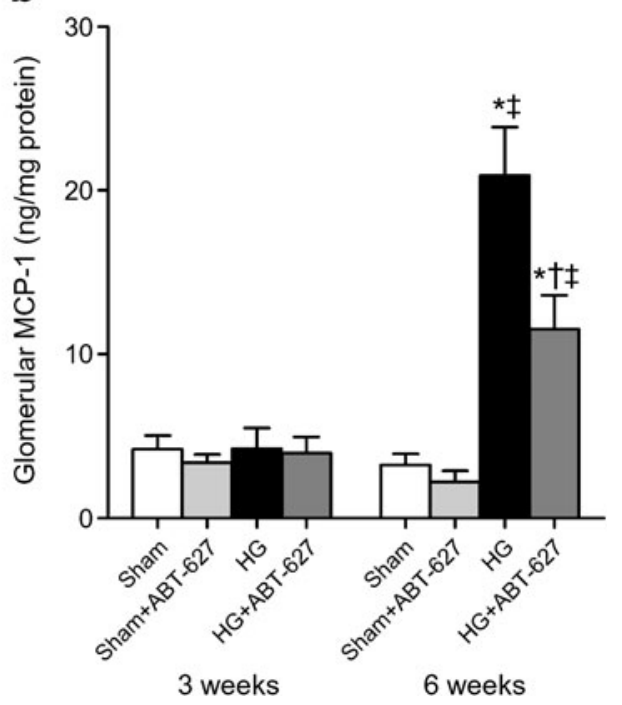




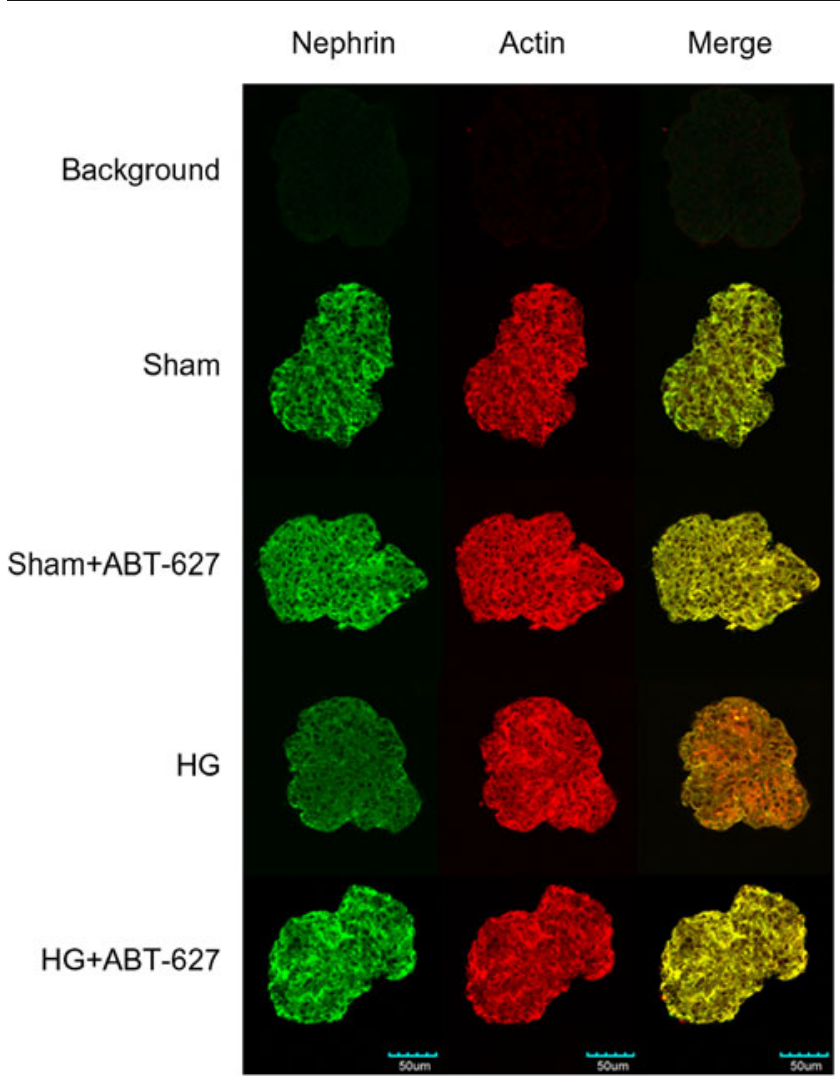

Fig. 5 Representative images of localisation of nephrin protein in the glomeruli of sham and hyperglycaemic (HG) untreated and ABT-627treated rats, as determined by immunofluorescence. All photographs were taken under the same conditions for the laser confocal microscope using a $\times 60$ lens and quantified by calculating the ratio to actin intensity using phalloidin staining; $n=4-5$ rats in each group. Negative controls for the primary nephrin antibody and the absence of phalloidin staining were evaluated as indicated above (Methods) and used to calculate the corrected intensity for nephrin and actin staining

that treatment with ABT-627, administered orally at $5 \mathrm{mg} \mathrm{kg}^{-1}$ day $^{-1}$ to hyperglycaemic rats, prevented increase in $\mathrm{P}_{\mathrm{alb}}$ in conjunction with reduction of proteinuria. The use of isolated glomeruli allowed us to measure the glomerular capillary $\mathrm{P}_{\mathrm{alb}}$ independently of the potential confounding effects of changes in mean arterial blood pressure and/or renal haemodynamics during measurement. Thus our findings demonstrate that a reduction in mean arterial BP or GFR is not a prerequisite for the anti-proteinuric effect of $\mathrm{ET}_{\mathrm{A}}$ blockade in the early phases of diabetic nephropathy. Collectively, these data are consistent with a nonhaemodynamic effect of endothelin-1 on glomerular filtration barrier function.

In patients with diabetic nephropathy, Wenzel et al. showed a reduction in macroalbuminuria after 12 weeks of treatment with the moderately selective $\mathrm{ET}_{\mathrm{A}}$ antagonist avosentan [22]. This effect was observed without a change in BP, suggesting a pressure-independent anti-proteinuric effect of this antagonist [22]. Most of those participants were already on a combination of treatment with angiotensin receptor blockers and ACE inhibitors, providing evidence that the anti-proteinuric effect of endothelin antagonism was independent of any effect on the reninangiotensin system. Opocenský et al. observed that lateonset $\mathrm{ET}_{\mathrm{A}}$ blockade reduces proteinuria in homozygous Ren-2 (also known as Ren2) rats despite severe hypertension [23]. Hocher and colleagues reported that the $\mathrm{ET}_{\mathrm{A}}$ antagonist, LU 135252, reduced proteinuria and completely normalised glomerular matrix protein expression in streptozotocin-induced hyperglycaemic rats [24].

In vitro experiments using isolated glomeruli from hyperglycaemic rats incubated with water-soluble endothelin peptide antagonists (BQ compounds) were conducted in order to investigate the specific role of glomerular $\mathrm{ET}_{\mathrm{A}}$ and $\mathrm{ET}_{\mathrm{B}}$ in permeability, independently of haemodynamic influence. $\mathrm{ET}_{\mathrm{A}}$ antagonism with $\mathrm{BQ}-123$ reduced the $\mathrm{P}_{\mathrm{alb}}$ defect of glomeruli from hyperglycaemic rats. The rapid effect of the antagonist on $\mathrm{P}_{\mathrm{alb}}$ can be explained by the role of $\mathrm{ET}_{\mathrm{A}}$ in rearrangement of the actin cytoskeleton in podocytes. Morigi et al. reported that shigatoxin stimulates the synthesis of endothelin-1, which may regulate cytoskeleton remodelling and thus glomerular permeability in an autocrine manner. Cytoskeleton rearrangement is associated with an increase in albumin permeability detected by transepithelial passage of fluorescent albumin to the basolateral compartment of podocytes [25]. It has been postulated that endothelin-1 released from glomerular endothelial cells or podocytes might interact with signalling to filtration-slit components and directly influence podocyte function [26]. Selective blockade of $\mathrm{ET}_{\mathrm{B}}$ via $\mathrm{BQ}-788 \mathrm{had}$ no effect on $\mathrm{P}_{\mathrm{alb}}$ of isolated glomeruli from hyperglycaemic rats. These results clearly suggest that $\mathrm{ET}_{\mathrm{B}}$ is unlikely to play a role in the regulation of glomerular permeability in hyperglycaemic glomeruli. We recently observed that exogenous endothelin-1 added to cultured podocytes increased $\mathrm{P}_{\mathrm{alb}}$ directly, an effect that can be blocked by an $\mathrm{ET}_{\mathrm{A}}$, but not an $\mathrm{ET}_{\mathrm{B}}$ antagonist [27]. Thus, we speculate that any effect of $\mathrm{ET}_{\mathrm{B}}$ on modulation of proteinuria is via haemodynamic changes rather than direct effects on the filtration barrier.

Further evidence of a direct influence of endothelin-1 on glomerular filtration barrier function comes from our observations that ABT-627 reduced nephrin excretion and glomerular nephrin levels in treated hyperglycaemic rats. In several animal models of diabetes, nephrin excretion is increased while glomerular levels are decreased [28], which is consistent with the current study. Additional evidence that endothelin-1 is an important contributor to nephrin loss comes from a report that media taken from cultured endothelial cells conditioned with sera from pre-eclampsia patients induced nephrin loss from human cultured podocytes, an effect that can be blocked by $\mathrm{ET}_{\mathrm{A}}$ blockade [29]. 


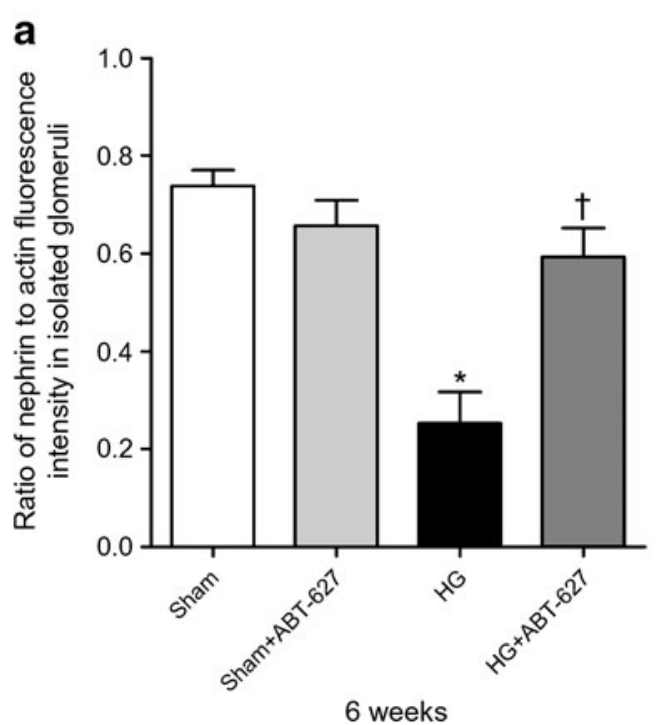

Fig. 6 a Effect of streptozotocin-induced hyperglycaemia (HG), with or without concurrent treatment with ABT-627, on glomerular nephrin levels as evaluated by immunofluorescence $(n=4-5$ rats in each group; $n=5-9$ glomeruli per rat), as well as on (b) nephrin excretion rate (nephrinuria; $n=5-6$ per group), both at the indicated times. a Two-way ANOVA resulted in the following $p$ values: Nephrin to actin ratio, 6 weeks, $p_{\mathrm{HG}}=0.0002, p_{\mathrm{ABT}-627}=0.0328, p_{\mathrm{HG} \times \mathrm{ABT}-627}=0.0017$.

Gagliardini et al. showed that avosentan prevented glomerular nephrin loss in a streptozotocin-induced model of diabetes, as detected via immunohistochemistry [13]. More recently, we reported that chronic endothelin-1 infusion in normoglycaemic rats for 2 weeks increased $\mathrm{P}_{\mathrm{alb}}$ without any change in BP and that incubation of isolated glomeruli with endothelin-1 for a little as 15 min significantly increased $\mathrm{P}_{\mathrm{alb}}$ [27]. Taken together, these studies all provide strong evidence that endothelin- 1 directly facilitates increased $\mathrm{P}_{\mathrm{alb}}$ within the glomerulus independently of haemodynamic effects.

We previously observed that $\mathrm{ET}_{\mathrm{A}}$ blockade with ABT627 reduced infiltration of macrophages and $\mathrm{T}$ cells in kidneys of hyperglycaemic rats after 10 weeks of treatment [12]. The current study used the same model to profile the relative time course of two early markers of inflammation, MCP-1 and soluble ICAM-1. After 6 weeks of hyperglycaemia induction, plasma and glomerular soluble ICAM-1 and MCP-1 concentrations were significantly increased, an increase largely prevented by ABT- 627 . There was no significant increase in inflammatory markers at the 3-week time-point. As we know from previous studies, infiltration of macrophages in the glomeruli and interstitium is one of the primary pathological features in models of diabetic nephropathy [30]. Leucocyte infiltration into inflammatory sites is mediated by coordinated actions of cell adhesion molecules and chemokines. Therefore our results support the hypothesis that endothelin-1, through the $\mathrm{ET}_{\mathrm{A}}$, functions as an early pro-inflammatory signal. This

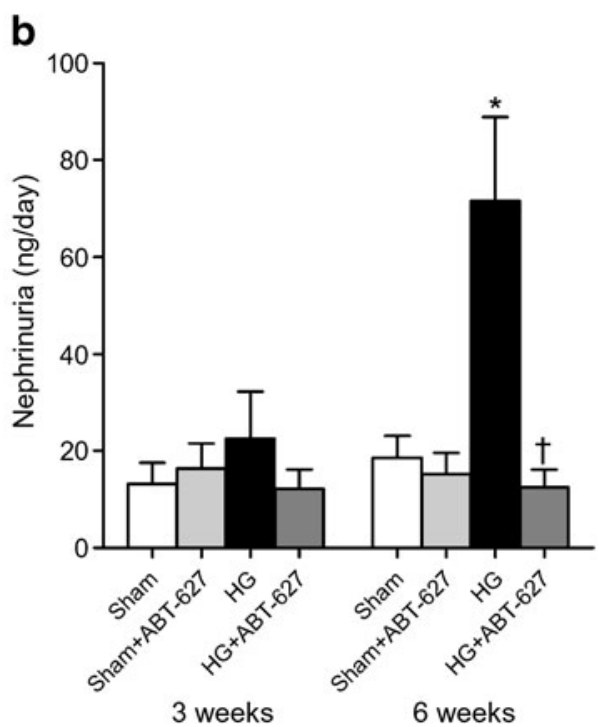

Post hoc analysis comparing individual means are denoted by ${ }^{*} p<0.05$ vs sham and ${ }^{\dagger} p<0.05$ vs hyperglycaemia. b Two-way ANOVA resulted in the following $p$ values: nephrinuria, 6 weeks, $p_{\mathrm{HG}}=0.0146, p_{\mathrm{ABT}-627}=0.0035, p_{\mathrm{HG} \times \mathrm{ABT}-627}=0.0078$. Post hoc analysis comparing individual means are denoted by $* p<0.05$ vs sham and ${ }^{\dagger} p<0.05$ vs hyperglycaemia

hypothesis is further supported by previous studies in other model systems showing that endothelin-1 can increase proinflammatory signalling pathways, such as mitogenactivated protein kinase and nuclear factor- $\mathrm{kB}$, which are known to increase production of chemoattractants and adhesion molecules such as MCP-1 and ICAM-1 [31].

ICAM-1 is a cell surface glycoprotein primarily involved in promoting leucocyte attachment to the endothelium and leucocyte transmigration through its expression on the vascular endothelium (diapedesis) and binding to $\beta 2$ leucocyte integrins [32]. ICAM-1 production can be induced by hyperglycaemia, advanced glycation endproducts, oxidative stress, hyperlipidaemia and hyperinsulinaemia [33]. Previous studies have shown that, in kidneys of patients with diabetic nephropathy, the accumulation of macrophages resulted from increased expression of cell adhesion molecules, such as ICAM-1 and selectins [34]. Several experimental models of diabetic nephropathy have investigated the correlation between ICAM-1 expression and disease activity. Sugimoto et al. reported that upregulation of ICAM-1 was associated with macrophage infiltration in early diabetic renal injury and was maintained during the period of study. The mechanism of ICAM-1 upregulation was mainly attributed to endothelial shear stress associated with elevated GFR in the hyperfiltration phase in hyperglycaemic rats [15]; these studies suggested that monoclonal antibodies against ICAM-1 abrogated the infiltration of macrophages in glomeruli in these hyperglycaemic rats. Similarly in ICAM-1-deficient $d b / d b$ mice, 
Chow et al. showed that ICAM-1 deficiency reduced glomerular macrophage infiltration with subsequent amelioration of glomerular hypertrophy and interstitial fibrosis [35], indicating a deteriorative mechanism for ICAM-1 in experimental models of diabetic nephropathy.

MCP-1, also termed chemokine C-C motif ligand 2, is a potent chemoattractant for monocytes/macrophages [36]. Numerous cell types, including tubular epithelial cells and mesangial cells, are known to be capable of producing MCP-1. As reported previously, MCP-1 production was upregulated in association with renal macrophage infiltration in diabetic patients [37]. The mechanisms leading to upregulation of MCP-1 in various types of renal injury, including diabetic nephropathy, are not fully understood. However, human and rodent mesangial cells can synthesise MCP-1 in response to several factors thought to be involved in glomerular injury. These include interleukin-1, TNF- $\alpha$ and low-density lipoprotein [38]. In the case of human mesangial cells, a high concentration of glucose and glycated albumin have been reported to promote MCP-1 production [39]. An additional influence that may induce synthesis of MCP-1 is the generation of reactive oxygen species [40]. MCP-1-deficiency in mice markedly reduced kidney macrophage accumulation and prevented the development of diabetic nephropathy [14]. Furthermore, blocking of the MCP-1/chemokine $\mathrm{C}-\mathrm{C}$ motif receptor 2 pathway ameliorated glomerulosclerosis, indicating that this pathway has an important role in progression of diabetic nephropathy [41].

Using a chronic infusion of endothelin-1 in the rat at doses that do not produce hypertension, we recently demonstrated a relationship between endothelin-1, soluble ICAM-1 and MCP-1 in the kidney [27]. Furthermore, endothelin-1 can increase expression of soluble ICAM-1 and MCP-1 in other tissue or cell types. In vitro studies suggest that endothelin-1 induces neutrophil adhesion to cardiac myocytes by increasing ICAM-1 expression, which is mediated via $\mathrm{ET}_{\mathrm{A}}$ on cardiac myocytes. ICAM-1 expression induced by activation of $\mathrm{ET}_{\mathrm{A}}$ appears to be mediated through the protein kinase $\mathrm{C}$ pathway [42]. Further studies using human brain-derived endothelial cells showed that in vitro endothelin-1 can directly stimulate $M C P-1$ mRNA expression and MCP-1 protein; and that this endothelin-1-induced MCP-1 production is mediated by the $\mathrm{ET}_{\mathrm{A}}[43]$.

Perspectives Our results support the hypothesis that $\mathrm{ET}_{\mathrm{A}}$ contributes to glomerular permeability defects and inflammation in hyperglycaemic rats. In a model of diabetic nephropathy, we have now shown that endothelin-1 induces nephrin loss, providing a mechanism for how $\mathrm{ET}_{\mathrm{A}}$ antagonists may produce changes in $\mathrm{P}_{\mathrm{alb}}$. These findings provide more thorough mechanistic support for the therapeutic potential of $\mathrm{ET}_{\mathrm{A}}$ antagonists in proteinuric renal disease as well as renal inflammation. Additional studies are needed to establish whether the beneficial effects of endothelin receptor blockade can reverse established changes in glomerular permeability, proteinuria and renal inflammation. Available endothelin-related antagonists include both $\mathrm{ET}_{\mathrm{A}}$ selective and combined $\mathrm{ET}_{\mathrm{A}}$ and $\mathrm{ET}_{\mathrm{B}}$ inhibitors. Given the often opposing actions of these subtypes, it will also be important to distinguish what, if any, receptor-specific effects occur on these clinically relevant variables.

Acknowledgements We would like to thank B. Molitoris, M. M. Kamocka and R. Sandoval for their assistance with nephrin immunostaining, confocal microscopy read-out and fluorescence data analyses. This work was supported by grants from the National Heart Lung and Blood Institute (HL69999 and HL64776) and a George M. O'Brien fellowship (to M. A. Saleh) at the Indiana Center for Biological Microscopy (NIH-funded). M. A. Saleh was also supported by an American Heart Association pre-doctoral fellowship and a grant from the government of Egypt.

Duality of interest The authors declare that there is no duality of interest associated with this manuscript.

\section{References}

1. Held PJ, Brunner F, Odaka M, Garcia JR, Port FK, Gaylin DS (1990) Five-year survival for end-stage renal disease patients in the United States, Europe, and Japan, 1982 to 1987. Am J Kidney Dis 15:451-457

2. Breyer JA, Bain RP, Evans JK et al (1996) Predictors of the progression of renal insufficiency in patients with insulindependent diabetes and overt diabetic nephropathy. The Collaborative Study Group. Kidney Int 50:1651-1658

3. Badr KF, Munger KA, Sugiura M, Snajdar RM, Schwartzberg M, Inagami $\mathrm{T}$ (1989) High and low affinity binding sites for endothelin on cultured rat glomerular mesangial cells. Biochem Biophys Res Commun 161:776-781

4. Rebibou JM, He CJ, Delarue F et al (1992) Functional endothelin 1 receptors on human glomerular podocytes and mesangial cells. Nephrol Dial Transplant 7:288-292

5. Tomita K, Nonoguchi H, Marumo F (1992) Regulation of $\mathrm{NaCl}$ transport by endothelin in renal tubules. Semin Nephrol 12:30-36

6. Firth JD, Ratcliffe PJ, Raine AE, Ledingham JG (1988) Endothelin: an important factor in acute renal failure? Lancet 2:1179-1182

7. Ruiz-Ortega M, Gomez-Garre D, Alcazar R et al (1994) Involvement of angiotensin II and endothelin in matrix protein production and renal sclerosis. J Hypertens Suppl 12:S51-S58

8. Hocher B, Lun A, Priem F, Neumayer HH, Raschack M (1998) Renal endothelin system in diabetes: comparison of angiotensinconverting enzyme inhibition and endothelin-A antagonism. J Cardiovasc Pharmacol 31(Suppl 1):S492-S495

9. Fukui M, Nakamura T, Ebihara I et al (1993) Gene expression for endothelins and their receptors in glomeruli of diabetic rats. J Lab Clin Med 122:149-156

10. Khan MA, Dashwood MR, Mumtaz FH, Thompson CS, Mikhailidis DP, Morgan RJ (1999) Upregulation of endothelin A receptor sites in the rabbit diabetic kidney: potential relevance to the early pathogenesis of diabetic nephropathy. Nephron 83:261-267 
11. Shin SJ, Hsiao PJ, Hsieh MC, Lee YJ, Tsai JH (1999) Increased urinary endothelin-1 excretion in newly diagnosed type 2 diabetic patients. Kaohsiung J Med Sci 15:589-596

12. Sasser JM, Sullivan JC, Hobbs JL et al (2007) Endothelin A receptor blockade reduces diabetic renal injury via an antiinflammatory mechanism. J Am Soc Nephrol 18:143-154

13. Gagliardini E, Corna D, Zoja C et al (2009) Unlike each drug alone, lisinopril if combined with avosentan promotes regression of renal lesions in experimental diabetes. Am J Physiol Renal Physiol 297:F1448-F1456

14. Chow FY, Nikolic-Paterson DJ, Ozols E, Atkins RC, Rollin BJ, Tesch GH (2006) Monocyte chemoattractant protein-1 promotes the development of diabetic renal injury in streptozotocin-treated mice. Kidney Int 69:73-80

15. Sugimoto H, Shikata K, Hirata K et al (1997) Increased expression of intercellular adhesion molecule-1 (ICAM-1) in diabetic rat glomeruli: glomerular hyperfiltration is a potential mechanism of ICAM-1 upregulation. Diabetes 46:2075-2081

16. Viberti GC, Messent J (1991) Risk factors for renal and cardiovascular disease in diabetic patients. Cardiology 79(Suppl 1): $55-61$

17. Langham RG, Kelly DJ, Cox AJ et al (2002) Proteinuria and the expression of the podocyte slit diaphragm protein, nephrin, in diabetic nephropathy: effects of angiotensin converting enzyme inhibition. Diabetologia 45:1572-1576

18. Baelde HJ, Eikmans M, Doran PP, Lappin DW, de Heer E, Bruijn JA (2004) Gene expression profiling in glomeruli from human kidneys with diabetic nephropathy. Am J Kidney Dis 43:636-650

19. Opgenorth TJ, Adler AL, Calzadilla SV et al (1996) Pharmacological characterization of A-127722: an orally active and highly potent ETA-selective receptor antagonist. J Pharmacol Exp Ther 276:473-481

20. Misra RP (1972) Isolation of glomeruli from mammalian kidneys by graded sieving. Am J Clin Pathol 58:135-139

21. Savin VJ, Sharma R, Lovell HB, Welling DJ (1992) Measurement of albumin reflection coefficient with isolated rat glomeruli. J Am Soc Nephrol 3:1260-1269

22. Wenzel RR, Littke T, Kuranoff S et al (2009) Avosentan reduces albumin excretion in diabetics with macroalbuminuria. J Am Soc Nephrol 20:655-664

23. Opocensky M, Kramer HJ, Backer A et al (2006) Late-onset endothelin-A receptor blockade reduces podocyte injury in homozygous Ren-2 rats despite severe hypertension. Hypertension 48:965-971

24. Hocher B, Schwarz A, Reinbacher D et al (2001) Effects of endothelin receptor antagonists on the progression of diabetic nephropathy. Nephron 87:161-169

25. Morigi M, Buelli S, Zanchi C et al (2006) Shigatoxin-induced endothelin-1 expression in cultured podocytes autocrinally mediates actin remodeling. Am J Pathol 169:1965-1975

26. Harita Y, Kurihara H, Kosako H et al (2009) Phosphorylation of nephrin triggers $\mathrm{Ca} 2+$ signaling by recruitment and activation of phospholipase C- $\gamma 1$. J Biol Chem 284:8951-8962
27. Saleh MA, Boesen EI, Pollock JS, Savin VJ, Pollock DM (2010) Endothelin-1 increases glomerular permeability and inflammation independent of blood pressure in the rat. Hypertension 56:942-949

28. Bonnet F, Cooper ME, Kawachi H, Allen TJ, Boner G, Cao Z (2001) Irbesartan normalises the deficiency in glomerular nephrin expression in a model of diabetes and hypertension. Diabetologia 44:874-877

29. Collino F, Bussolati B, Gerbaudo E et al (2008) Preeclamptic sera induce nephrin shedding from podocytes through endothelin-1 release by endothelial glomerular cells. Am J Physiol Renal Physiol 294:F1185-F1194

30. Shikata K, Makino H (2001) Role of macrophages in the pathogenesis of diabetic nephropathy. Contrib Nephrol 134:46-54

31. Luft FC, Mervaala E, Muller DN et al (1999) Hypertensioninduced end-organ damage: a new transgenic approach to an old problem. Hypertension 33:212-218

32. Rothlein R, Wegner C (1992) Role of intercellular adhesion molecule-1 in the inflammatory response. Kidney Int 41:617-619

33. Galkina E, Ley K (2006) Leukocyte recruitment and vascular injury in diabetic nephropathy. J Am Soc Nephrol 17:368-377

34. Hirata K, Shikata K, Matsuda M et al (1998) Increased expression of selectins in kidneys of patients with diabetic nephropathy. Diabetologia 41:185-192

35. Chow FY, Nikolic-Paterson DJ, Ozols E, Atkins RC, Tesch GH (2005) Intercellular adhesion molecule-1 deficiency is protective against nephropathy in type 2 diabetic $\mathrm{db} / \mathrm{db}$ mice. J Am Soc Nephrol 16:1711-1722

36. Luster AD (1998) Chemokines-chemotactic cytokines that mediate inflammation. N Engl J Med 338:436-445

37. Wada T, Furuichi K, Sakai N et al (2000) Up-regulation of monocyte chemoattractant protein-1 in tubulointerstitial lesions of human diabetic nephropathy. Kidney Int 58:1492-1499

38. Rovin BH, Tan LC (1993) LDL stimulates mesangial fibronectin production and chemoattractant expression. Kidney Int 43:218-225

39. Ihm CG, Park JK, Hong SP et al (1998) A high glucose concentration stimulates the expression of monocyte chemotactic peptide 1 in human mesangial cells. Nephron 79:33-37

40. Schena FP, Gesualdo L, Grandaliano G, Montinaro V (1997) Progression of renal damage in human glomerulonephritides: is there sleight of hand in winning the game? Kidney Int 52:1439-1457

41. Kanamori H, Matsubara T, Mima A et al (2007) Inhibition of MCP-1/CCR2 pathway ameliorates the development of diabetic nephropathy. Biochem Biophys Res Commun 360:772-777

42. Hayasaki Y, Nakajima M, Kitano Y, Iwasaki T, Shimamura T, Iwaki K (1996) ICAM-1 expression on cardiac myocytes and aortic endothelial cells via their specific endothelin receptor subtype. Biochem Biophys Res Commun 229:817-824

43. Chen P, Shibata M, Zidovetzki R, Fisher M, Zlokovic BV, Hofman FM (2001) Endothelin-1 and monocyte chemoattractant protein-1 modulation in ischemia and human brain-derived endothelial cell cultures. J Neuroimmunol 116:62-73 